\title{
Analysis and Design of Power Electronic Transformer based Power Quality Improvement
}

\author{
B .T. Kalyan ${ }^{1}$, P. Ram Prasad ${ }^{2}$ \\ ${ }^{I}$ (Power Electronic Systems, Department of EEE, Swarnandhra College of engineering, jntu kakinada) \\ ${ }^{2}$ (Associate Professor, Department of EEE, Swarnandhra College of engineering, Narasapur)
}

\begin{abstract}
A new type of transformer based on Power Electronics (PET) has been proposed in this paper, which realizes voltage transformation, galvanic isolation, and power quality improvements in a single device. The PET provides a fundamentally different and more complete approach in transformer design by using power electronics on the primary and secondary sides of the transformer. Several features such as instantaneous voltage regulation, voltage sag compensation and power factor correction can be combined into PET. This paper presents a novel topology of power electronic transformer. In the design process, the AC/DC, DC/AC, $A C / A C$ converters and high frequency transformer have been used. One matrix converter operates as AC/AC converter in power electronic transformer. The designed power electronic transformer performs typical functions and has advantages such as power factor correction, voltage sag and swell elimination, voltage flicker reduction and protection capability in fault situations. The proposed power electronic transformer has been modeled using MATLAB/SIMULINK and Power quality improvement with proposed model has been verified by the simulation results.
\end{abstract}

Keywords- Power electronic transformer; AC/AC Matrix converter; Power Quality; High Frequency Transformer; Space vector Technique

\section{Introduction}

Transformers are widely used in electric power system to perform the primary functions, such as voltage transformation and isolation. Transformers are one of the heaviest and most expensive devices in an electrical system because of the large iron cores and heavy copper windings in the composition [1].

A new type of transformers based on Power Electronics (PET) has been introduced, which realizes voltage transformation, galvanic isolation, and power quality improvements in a single device. The PET provides a fundamentally different and more complete approach in transformer design by using power electronics on the primary and secondary sides of the transformer. Several Features such as instantaneous voltage regulation, voltage sag compensation and power factor correction can be combined into PET.

Different topologies have been presented for realizing the PET, in recent years [2]-[7]. In [2] the $\mathrm{AC} / \mathrm{AC}$ buck converter has been proposed to transform the voltage level directly and without any isolation transformer. This method would cause the semiconductor devices to carry very high stress.

In second type, the line side AC waveform is modulated into a High or medium Frequency (HF or MF) square wave, coupled to the secondary of HF (MF) transformer, and again is demodulated to AC form by a converter in second side of HF (MF) transformer. This method however does not provide any benefits such as instantaneous voltage regulation and voltage sag compensation due to lack of energy storage system. In second type matrix converter is a direct AC-AC power converter employing bidirectional switches. In addition to the basic ability of power converter providing a sinusoidal variable voltage variable frequency to the load, matrix converter has many attractive features: no bulky DC-link capacitor, ability to make sinusoidal input current, high efficiency, compact circuit design and regeneration capability [3]-[5].

Another type is a three-part design that utilizes an input stage, an isolation stage, and an output stage [6]-[9]. These types enhance the flexibility and functionality of the electronic transformers owing to the available DC links. This approach can perform different power quality functions and provide galvanic isolation but they need whether too many power electronic converters and DC-link electrolytic capacitors. Thus they result in a rather cumbersome solution. Custom power devices are introduced in the distribution system to deal with various power quality problems faced by industrial and commercial customers due to increase insensitive loads such as computer and adjustable speed drives and use of programmable logic control in the industrial process. This paper investigates the PET that includes three parts: input stage, an isolation stage, and an output stage. Proposed PET includes AC/AC matrix converter.

The proposed AC/AC converter can generate desired output voltage from square input voltage. The main point of proposed PET is reduction of the stage and components of the three-part PETs. The reliability and power quality of distribution system can be significantly improved by using proposed PET. To verify the performance of the proposed PET, computer-aided simulations are carried out using MATLAB/SIMULINK. 


\section{Conventional Pets}

Fig. 1 shows the basic block diagram of the PET using HF (MF) AC-link without DC-link capacitor. In this system, the line side AC waveform is modulated with a converter to a high-frequency square-wave and passed through a HF (MF) transformer and again with a converter, it is demodulated to AC form powerfrequency. Since the transformer size is inversely proportional to the frequency, the HF (MF) transformer will be much smaller than the power-frequency transformer. So, the transformer size, weight and stress factors reduced considerably [3].

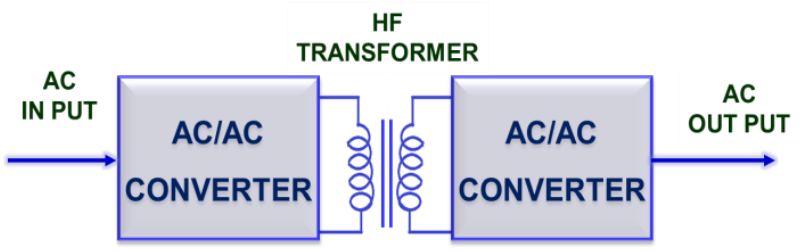

Figure 1 Block Diagram of electronic transformer using High Frequency AC link

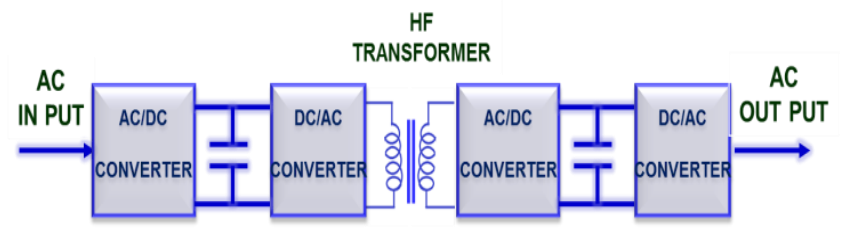

Figure 2 Block Diagram of Power Electronic based Transformer (PET) with DC link

This converter does not provide any benefits in terms of protect the critical loads from the instantaneous power interruptions due to lack of energy storage system [7].Fig. 2 shows the basic block diagram of a PET with DC link capacitor which includes three stages. First stage is an AC/DC converter which is utilized to shape the input current, to correct the input power factor, and to regulate the voltage of primary DC bus. Second stage is an isolation stage which provides the galvanic isolation between the primary and secondary side. In the isolation stage, the DC voltage is converted to a high-frequency square wave voltage, coupled to the secondary of the HF (MF) transformer and is rectified to form the DC link voltage. The output stage is a voltage source inverter which produces the desired AC waveforms [4]-[9].

In comparison to first PET, the voltage or current of PET can be flexibly controlled in either side of HF (MF) transformer. It is possible to add energy storage to enhance the ride-through capability of the PET or to prepare integrated interface for distributed resources due to the available DC links. It prevents the voltage or current harmonics to propagate in either side of the transformer, even if the input voltage has low order harmonic content or the load is not linear but they need too many converters (AC/DC or DC/AC) and DC-link electrolytic capacitors. Thus they are resulted in a rather cumbersome solution and multiple power conversion stages can lower the transformer efficiency.

\section{Proposed Pet}

The block diagram of the proposed PET is shown in Fig. 3. As can be seen from the Fig. 3, this is a three-stage design that includes an input stage, an isolation stage and an output stage. In the input stage, there is a converter, which converts the input AC voltage to DC voltage. The second part of the converter is formed by a DC/AC converter. This part of the converter contains the MF transformer with the high insulation capability. In the output part, the high frequency voltage is revealed as a power-frequency voltage. In this paper, a three part design is introduced. It is a new configuration based on the matrix converter with new function shown in Fig. 3. It can provide desired output voltage. In addition, it performs power quality functions, such as sag correction, reactive power compensation and is capable to provide three-phase power from a single phase system. The PET has three stages and each stage can be controlled independently from the other one. Many advantages of the PET such as output power quality and power factor correction depend on appropriate close-loop control, and correlative research is necessary. The reliability of a system is indirectly proportional to the number of its components. The main purpose of proposed PET is reduction of the power delivery stage (AC/DC and DC/AC links) in PET with DC-link.

The input stage is a three or single phase PWM rectifier, which is used to convert the primary low frequency voltage into the DC voltage. The main functions associated with the rectifier control are shaping the input current, controlling the input power factor, and keeping the DC-link voltage at the desired reference value. Many control methods are presented for control of input stage in conventional PET. Fig. 4 shows three phase rectifier with input inductances. A three phase PWM rectifier is used in thispaper, which operates same as input stage of conventional PET [8]-[9]. As can be seen from Fig. 5, the reference for the active current is derived 
from the DC voltage outer loop. The reference for the reactive current is set to zero to get unity power factor. The current error signals are input the current regulators and then form the modulation signals. If the $d$ axis of the reference frame is aligned to the grid voltage, we obtain Vinq $=0$.

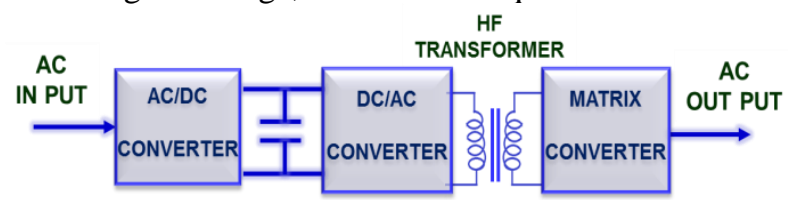

Figure 3 Block Diagram of proposed PET with DC link

Isolation stage is contained a single-phase high frequency voltage source converter (VSC), which converts the input DC voltage to AC square voltage with high (or medium) frequency and HF (MF) transformer. The main functions of the HF (MF) transformer are such as voltage transformation and isolation between source and load.

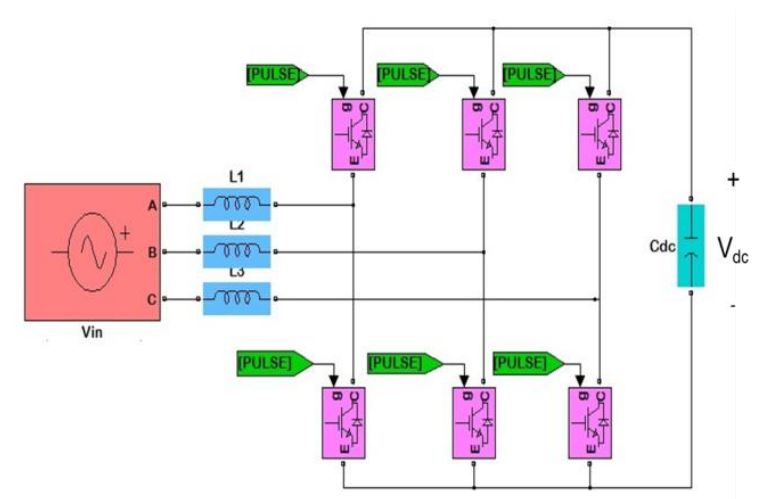

Figure 4 Structure of the proposed input stage

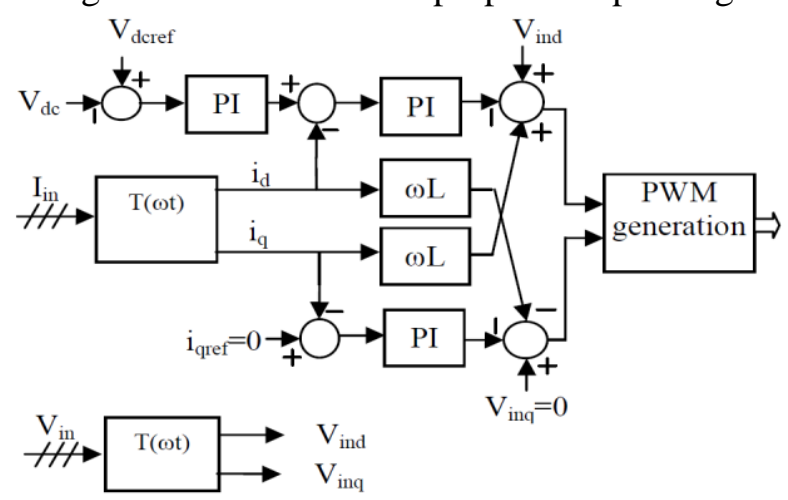

Figure 5 Input stage control diagram

Structure of the proposed isolation stage is shown in Fig.6 Circuit diagram of VSC is the same as Hbridge cell. The principle of modulation is based on a comparison of a sinusoidal reference waveform with zero carrier waveform.

The principle of switching H-bridge is described with conditions bellow:

Condition 1: if sin wave $\geq 0$, then $\mathrm{H} 1$ and $\mathrm{H} 2$ are turned on.

Condition 2: if sin wave $<0$, then $\mathrm{H} 3$ and $\mathrm{H} 4$ are turned on.

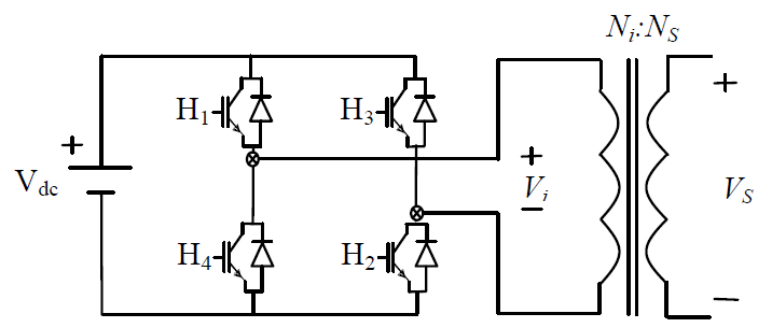

Figure 6 Structure of the proposed isolation stage 
Fig. 7 shows a matrix converter with novel function for square to sinusoidal voltage converter. Matrix converter topology employs six bidirectional switches to convert high frequency single-phase input directly to a power frequency $(50 / 60 \mathrm{~Hz})$ three- phase output.

The proposed converter generates desired output voltage with suitable shape and frequency. Several modulation strategies have been proposed for traditional inverters. Among these methods, space vector pulse width modulation (SVM) became a standard for the switching power converters. which has some advantages, such as immediate comprehension of the required commutation processes, simplified control algorithm, and maximum voltage transfer ratio without adding third harmonic components. They provided both mathematical treatment and a physical description and understanding of the drive transients even in the cases when machines are fed through electronic converters.

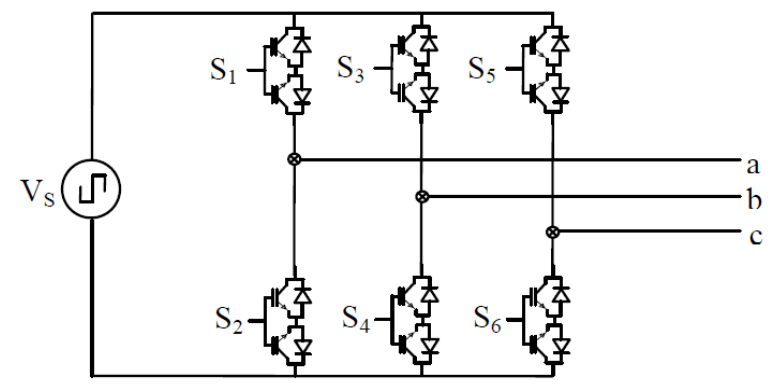

Figure 7 Proposed matrix converter

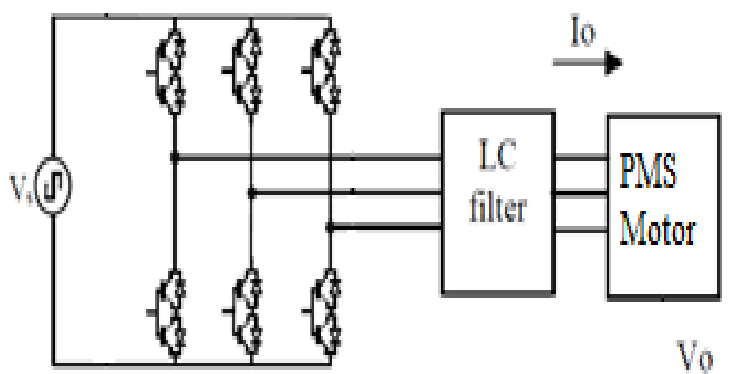

Figure 8 Circuit of output stage

Space Vector Pulse Width Modulation identifies each switching state of a two- or multilevel converter as a point in complex space. Then a reference phasor rotating in the plane at the fundamental frequency is sampled within each switching period, and the nearest three converter switched states are selected with duty cycles calculated to achieve the same volt-second average as the sampled reference phase. This directly controls the converter line-to-line voltages, and only implicitly develops the phase leg voltages an analytical expression is derived for the optimal apportioning factor that results in minimum THD[10]-[11].Comparing with pulse width modulation space vector modulation has more switching paterns.In proposed PET, space vector modulation technique applied to a matrix converter is employed. The main point of switching is, with changing of polarity in input sources on switches are turned off and other switches in arms are turned on.

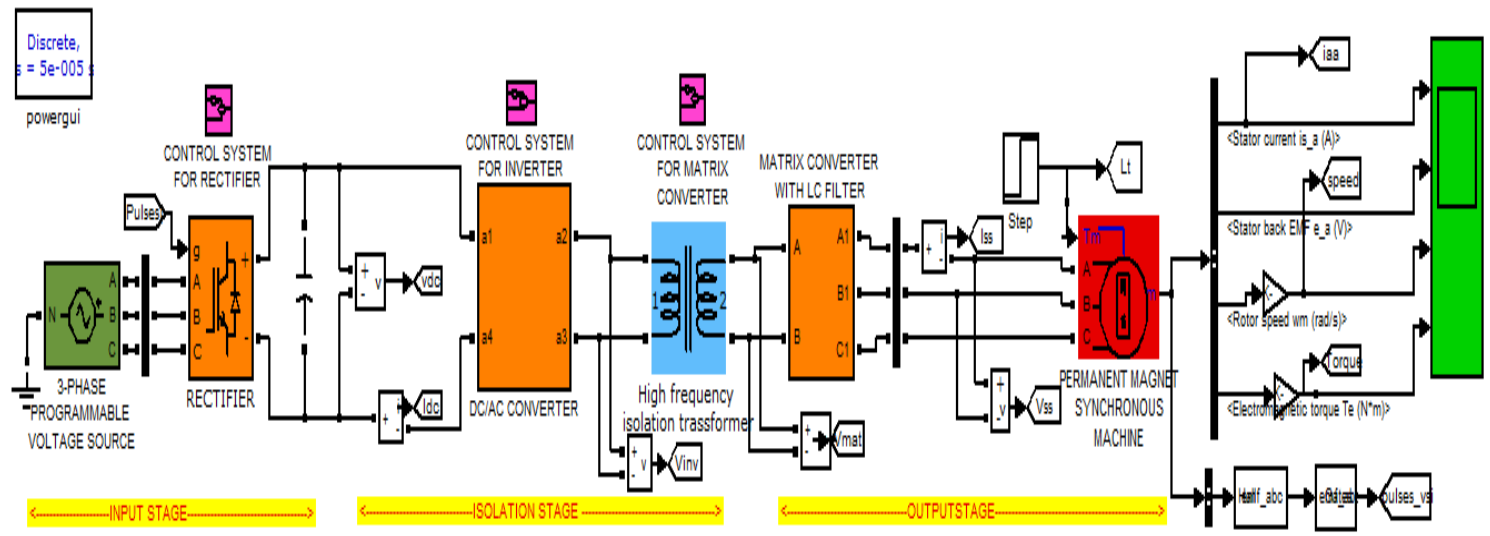

Fig 9. MATLAB / SIMULINK MODELING OF PROPOSED POWER ELECTRONIC TRANSFORMER 
In comparison with conventional PET with DC-link, in proposed converter power delivery stages and power electronic converters have been reduced and AC/AC matrix converter is used replaced by two (rectifier and inverter). This idea leads to the loss reduction, by processing the power in one stage instead of two stages.

Fig.8 shows the output stage with LC filter and permanent magnet synchronous machine [12].Comparing with conventional transformer with power electronic transformers has advantages as instantaneous voltage regulation, voltage sag compensation and power factor correction in PET without any external devices. The reliability and power quality of distribution system can be significantly improved by using proposed PET.

In the fig 9 shows MATLAB / SIMULINK modeling of proposed power electronic transformer. At the output stage a permanent magnet synchronous motor is connected. For voltage sag and Swell the output and speed torque of synchronous machine are at constant.

\section{Simulation Results}

To evaluate the expected performance of the PET, the design was simulated to predict steady state performance. A prototype based on the proposed topology is simulated using MATLAB/SIMULINK. Operation of proposed PET is described by Fig. 10. Fig. 10(a) shows input line voltage of PET. As it can be seen in Fig. 10(b), the DC-link voltage of input stage is $6800 \mathrm{~V}$. Fig. 10(c) depicts the output voltage of VSC in isolation stage that transforms DC voltage to medium frequency AC voltage as the transformer primary voltage. In the output stage, the medium frequency voltage is revealed as a $50 \mathrm{~Hz}$ waveform by AC/AC matrix converter, and synchronous machine in connected after the filter 10(f) shows rotor current, speed and torque of the Permanent Magnet Synchronous machine.

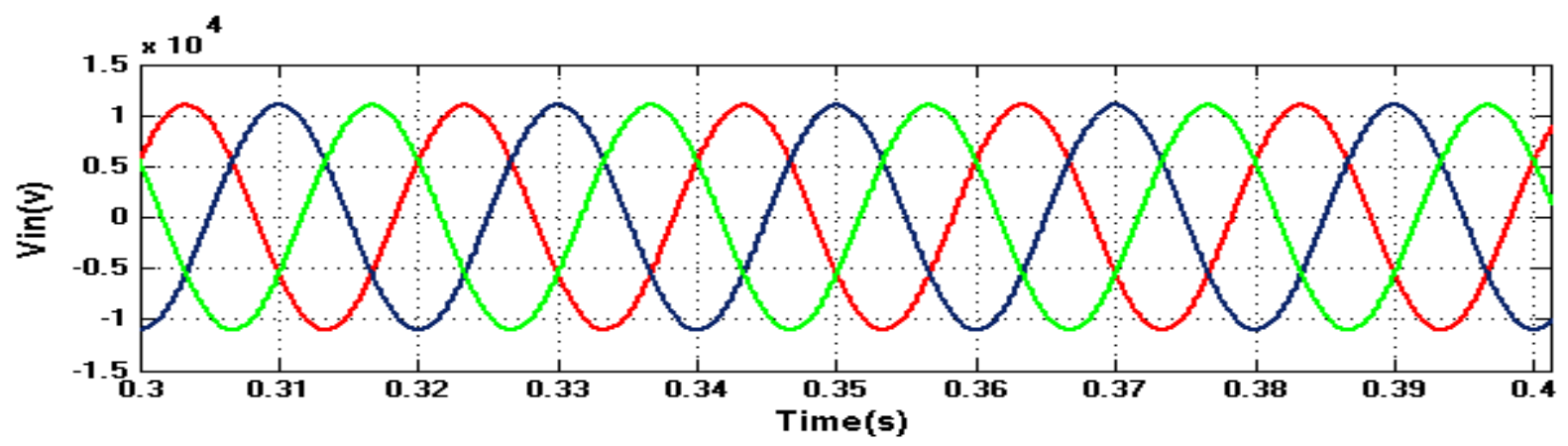

(a)

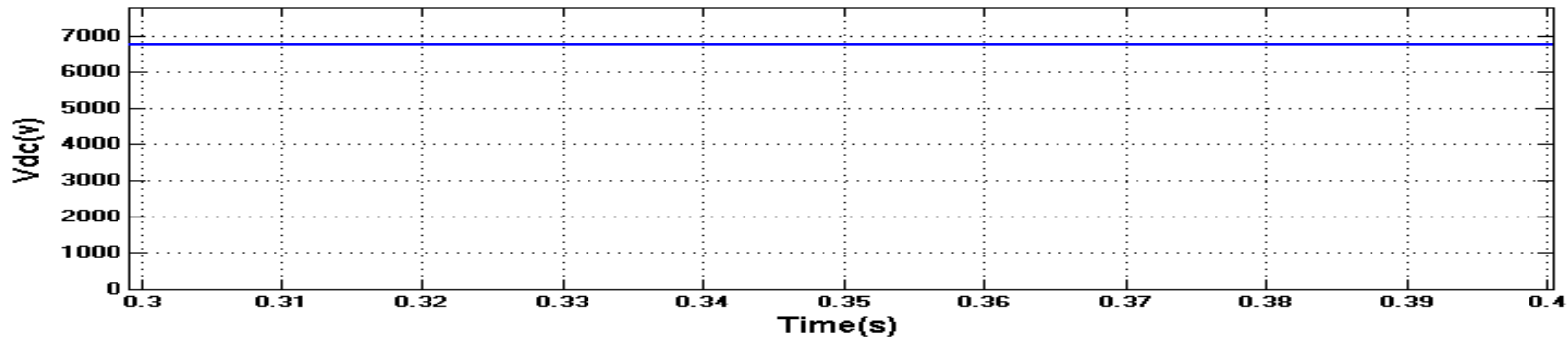

(b)

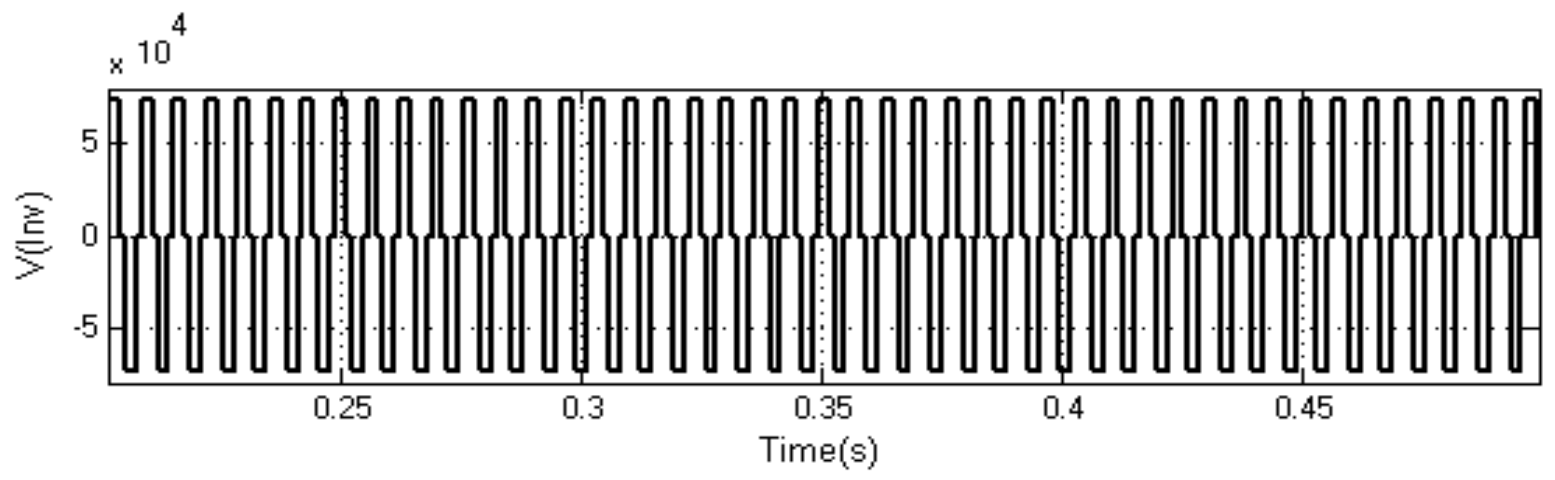

(c) 


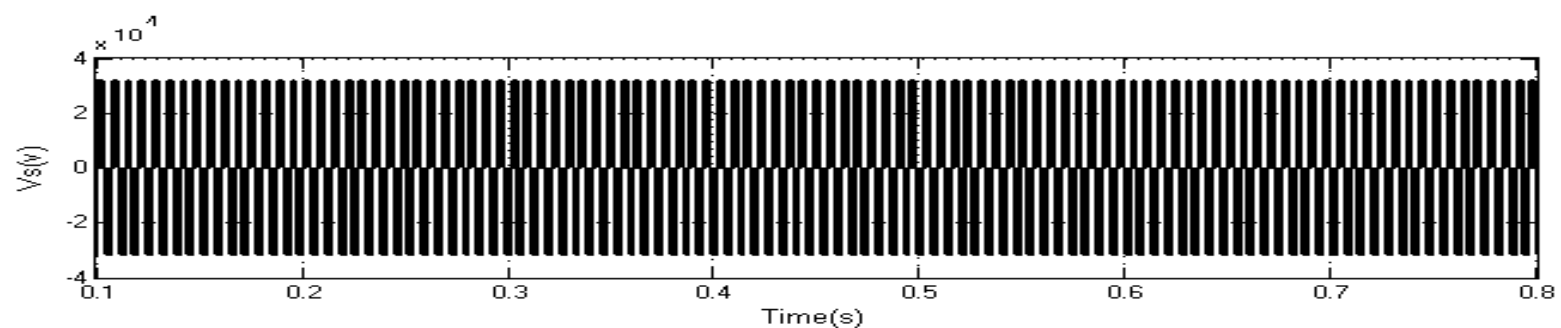

(d)

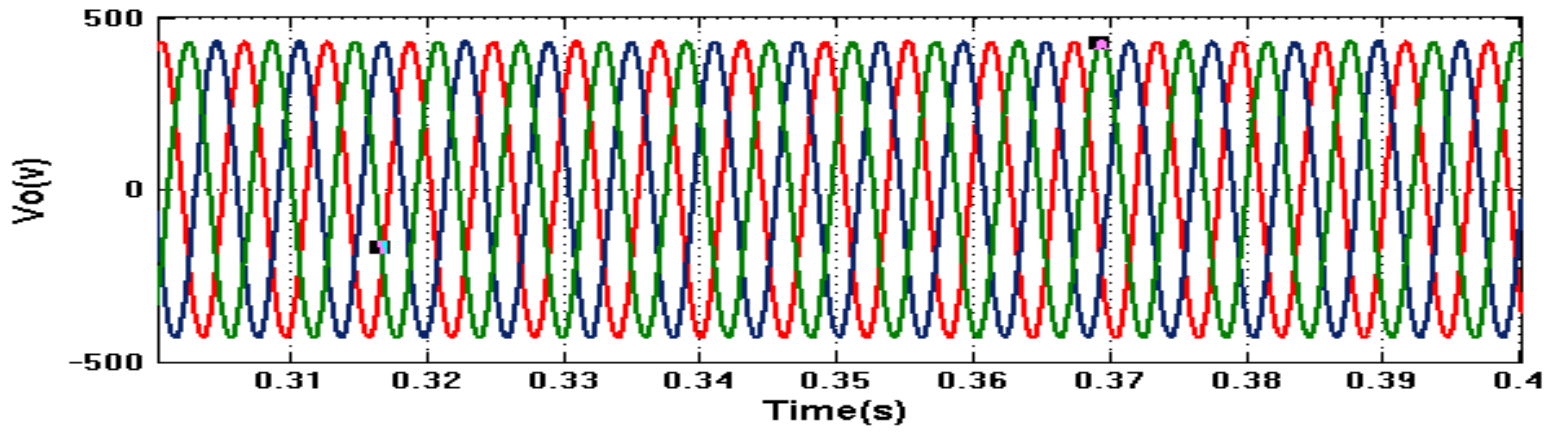

(e)

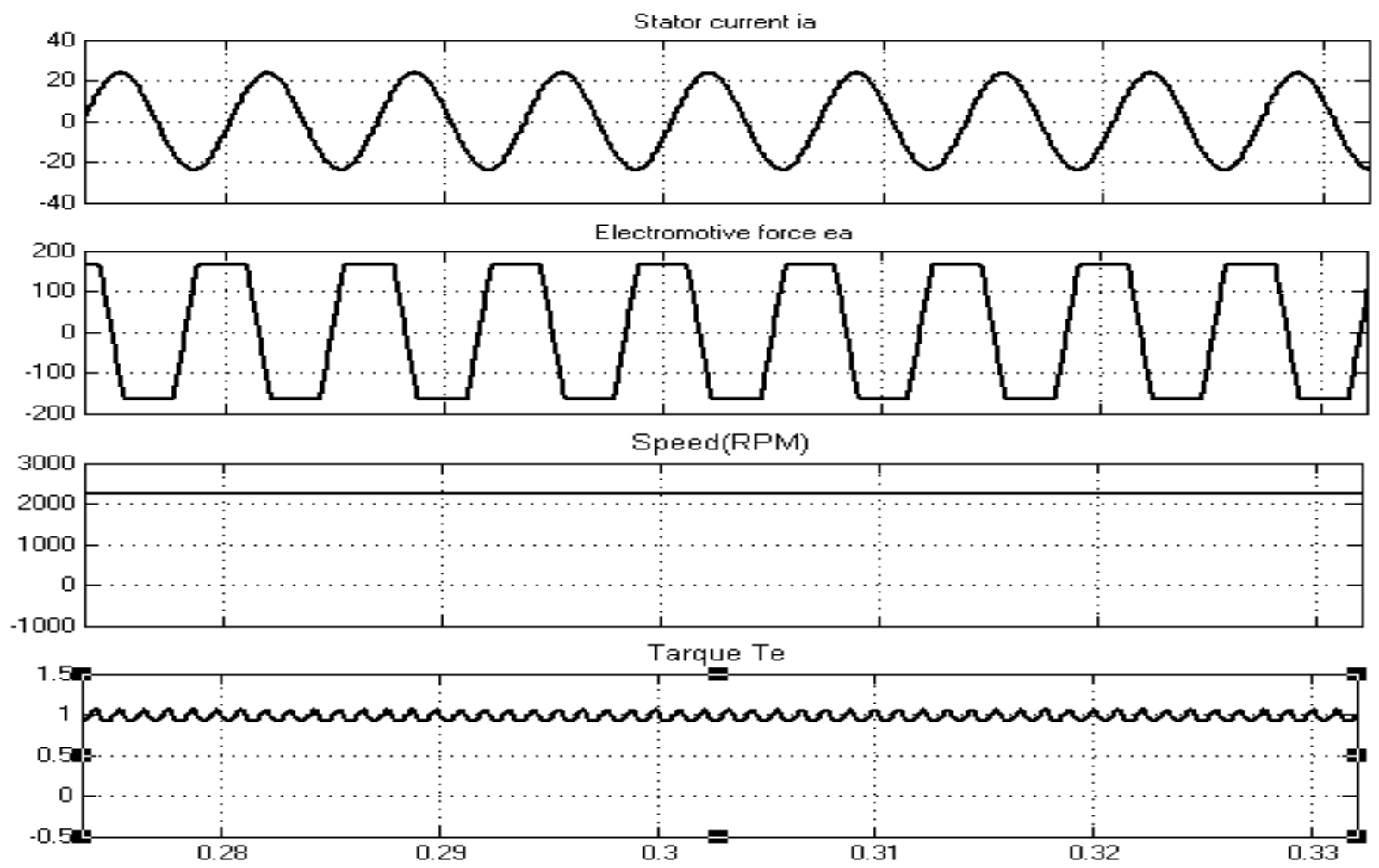

(f)

Figure 10. (a) Input voltage (b) DC-link voltage (c) MF transformer primary voltage (d) MF transformer secondary voltage (e) output voltage and (f) output of synchronous machine. 


\section{TABLE-I}

\begin{tabular}{|l|l|}
\hline Parameters & Value \\
\hline Input phase-phase voltage & $11 \mathrm{KV}$ \\
\hline Power frequency & $50 \mathrm{~Hz}$ \\
\hline MF transformer & $\begin{array}{l}10: 1, \quad 1000 \quad \mathrm{~Hz}, 30 \\
\mathrm{kVA}\end{array}$ \\
\hline Output voltage & $415 \mathrm{~V}$ \\
\hline $\begin{array}{l}\text { Matrix converter switching } \\
\text { frequency }\end{array}$ & $2050 \mathrm{~Hz}$ \\
\hline Load & $20 \mathrm{~kW}+\mathrm{j} 10 \mathrm{kVAR}$ \\
\hline LC filter & $2 \mathrm{mH}, 220 \mu \mathrm{F}$ \\
\hline $\mathrm{L}, \mathrm{Cdc}$ & $3 \mathrm{mH}, 2000 \mu \mathrm{F}$ \\
\hline
\end{tabular}

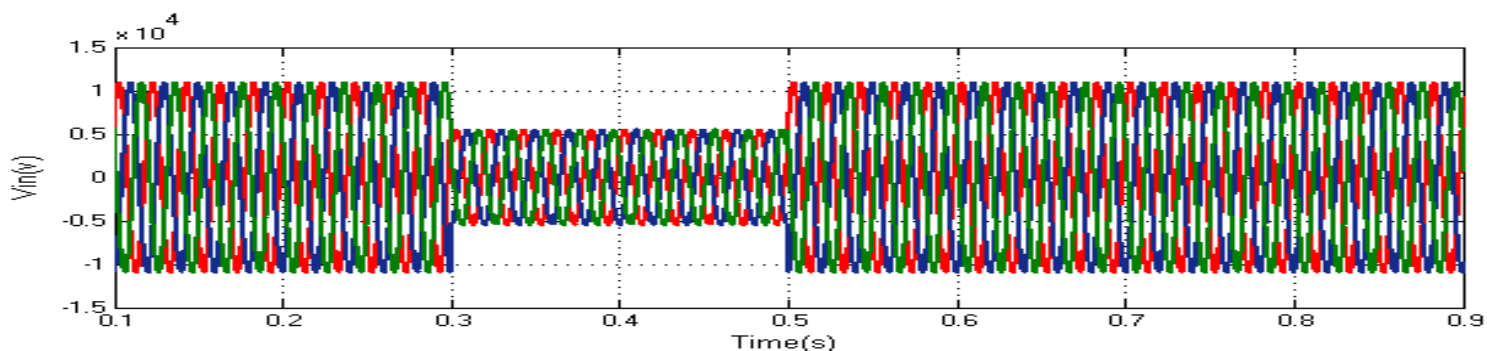

(a)

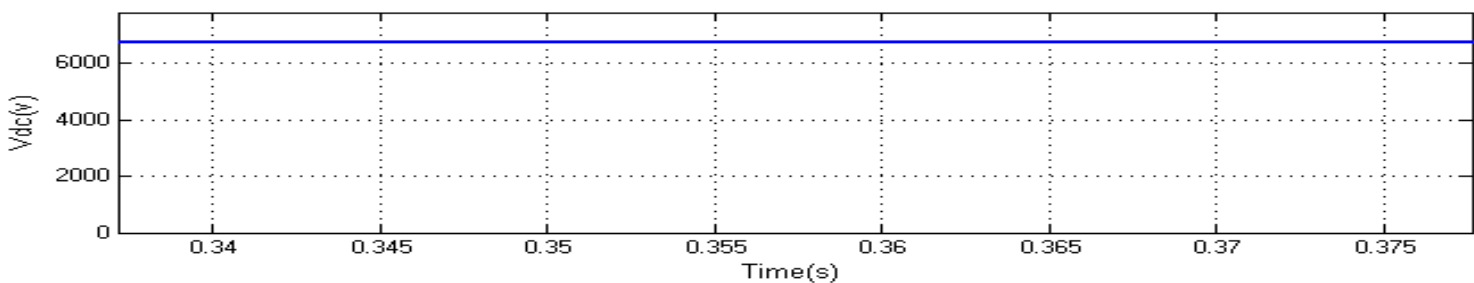

(b)

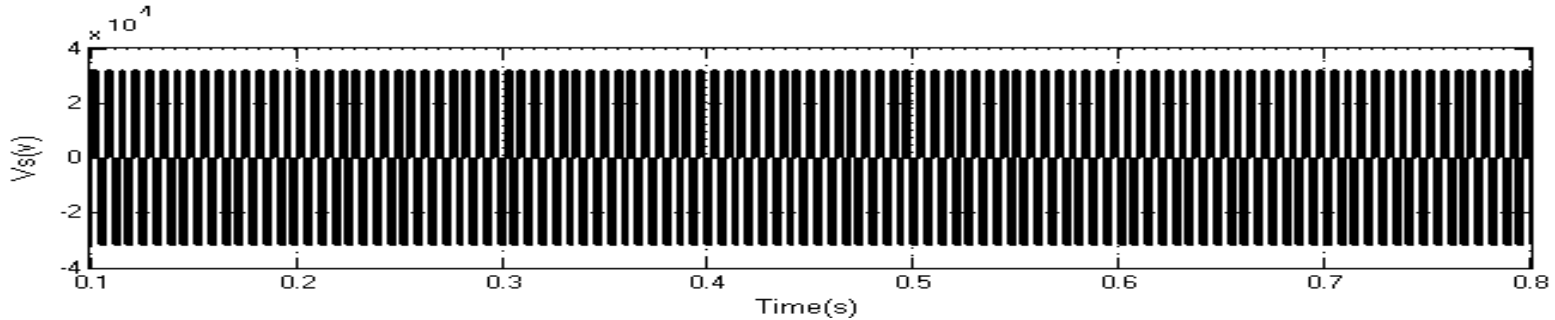

(c)

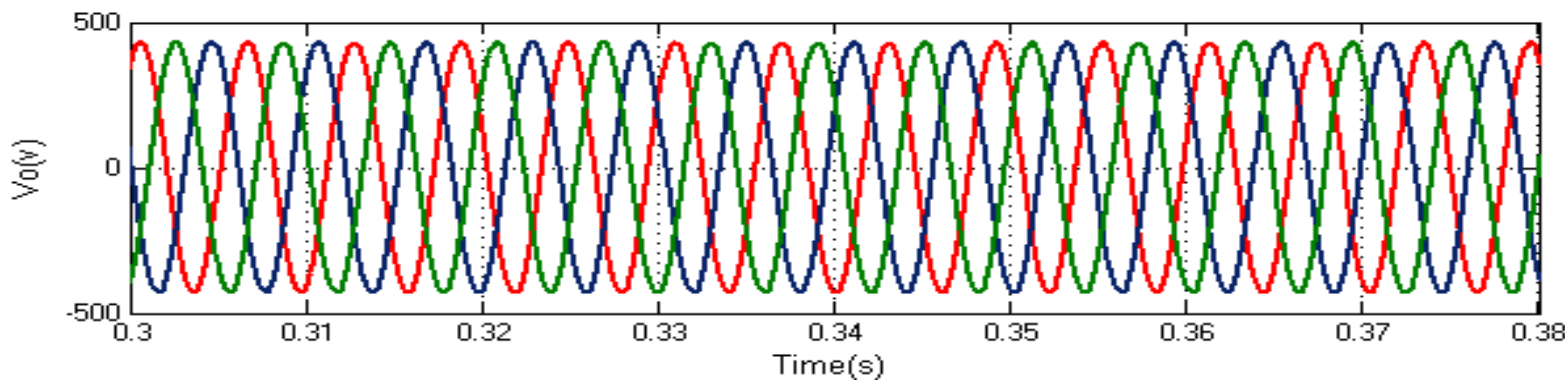

(d)

Figure 11. (a) Input voltage sag (b) DC-link voltage(c) Load voltage before filter and (d) load voltage 


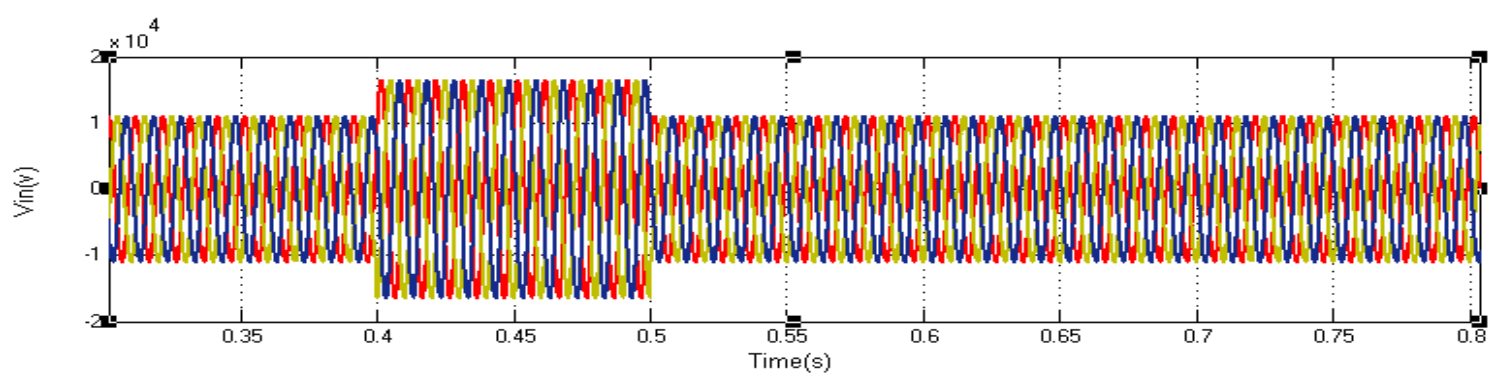

(a)

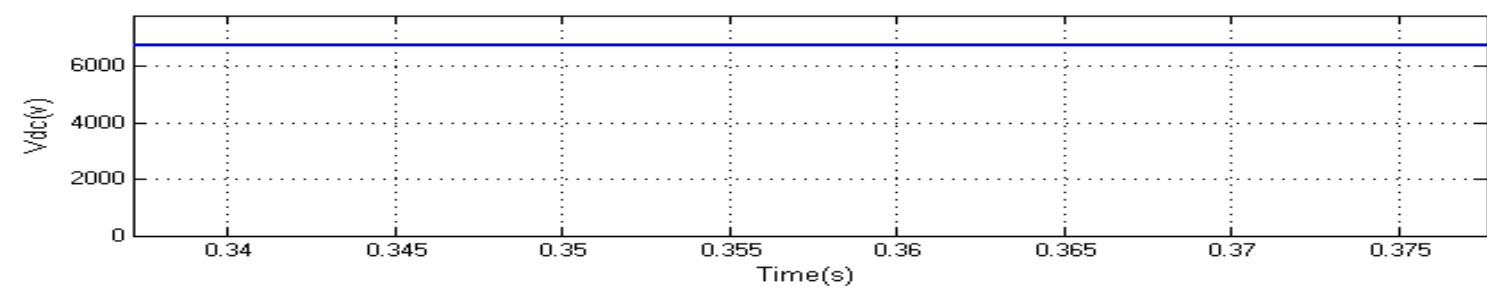

(b)

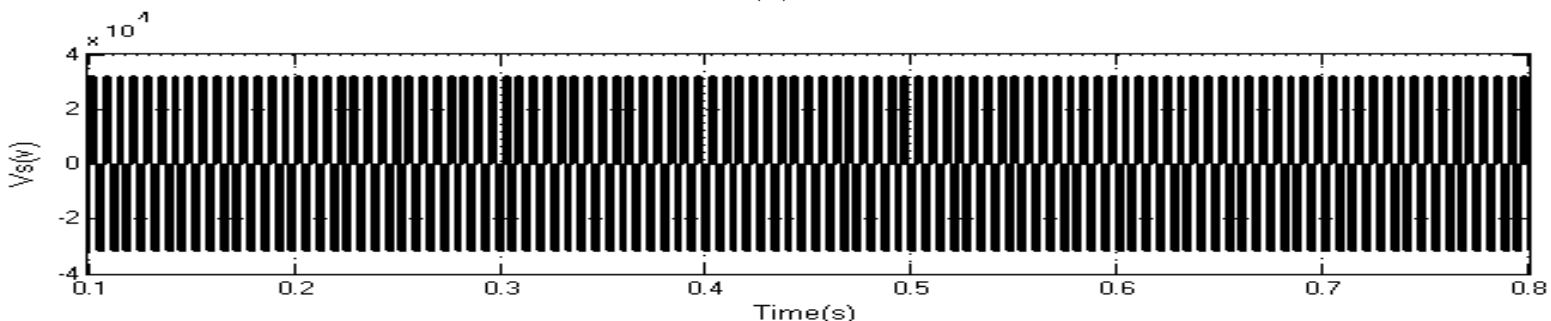

(c)

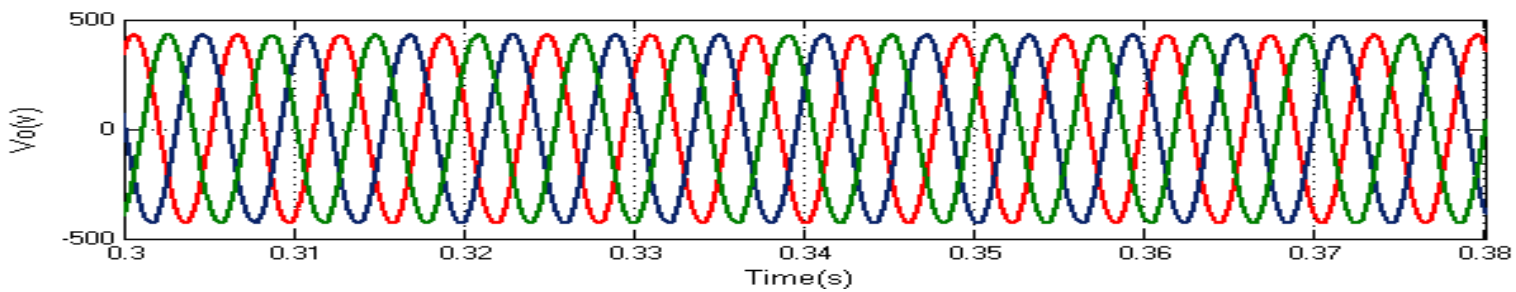

(d)

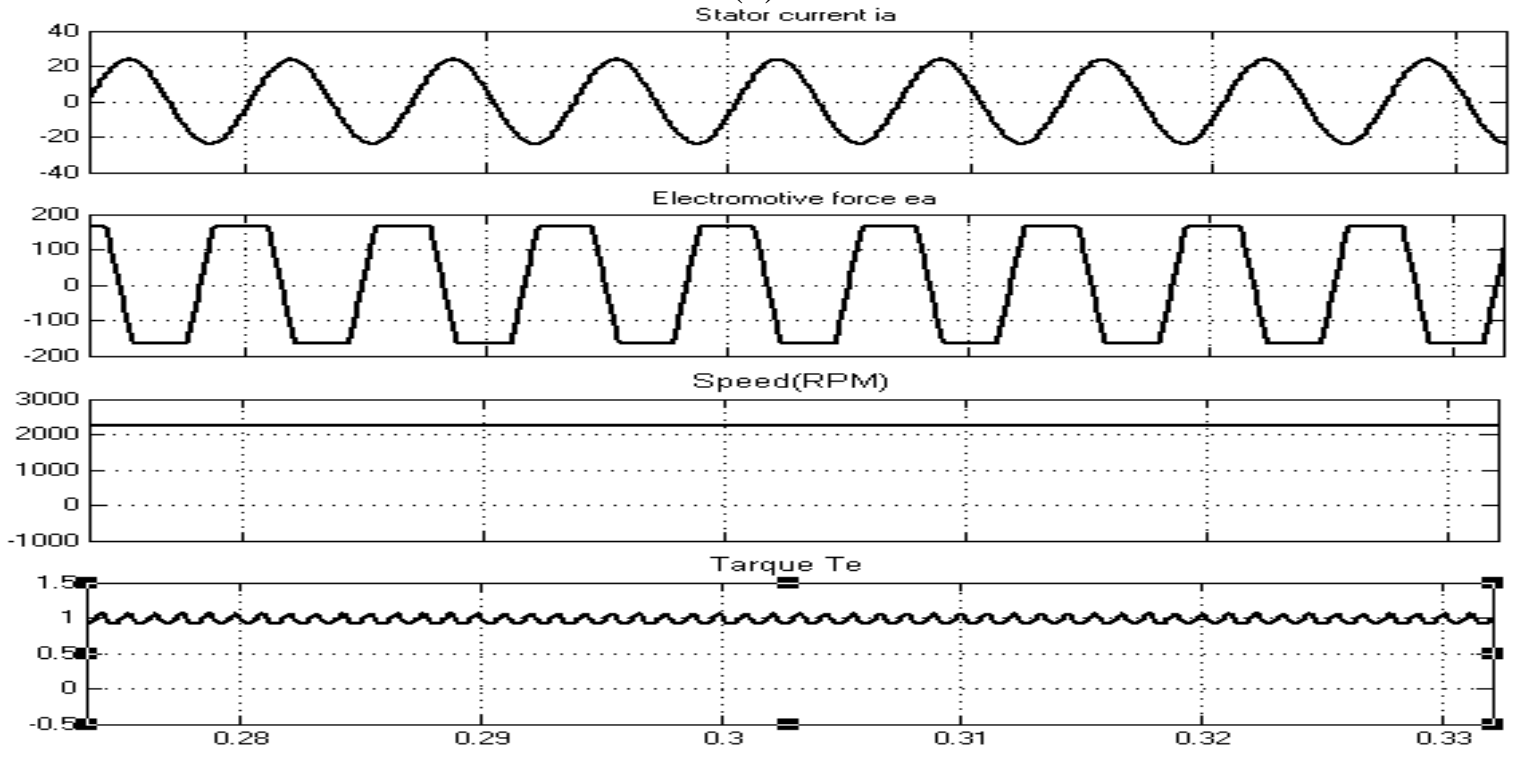

(e) 
Figure 12. (a) Input voltage swells (b) DC-link voltage (c) Load voltage before filter and (d) load voltage. (e) Torque speed characteristics of synchronous machine when sag $\&$ swell occurred

\section{Conclusion}

In this paper a new configuration of power electronic transformer with DC-Link capacitor has been proposed. To obtain higher efficiency, the AC/DC and DC/AC converters have been integrated in one converter. The topology described in this paper has many advantages such as power factor correction, voltage regulation, voltage sag and swell elimination, voltage flicker reduction. In proposed PET one AC/AC matrix converter is used to replace two converters and switching of matrix converter is easy and not complex. Simulation results showed some of advantages in proposed PET.

\section{References}

[1] M. Kang, P.N. Enjeti, I.J. Pitel, “Analysis and design of electronictransformers for electric power distribution system,” IEEE Trans.On Power Electronics, vol. 14, no. 6, pp. 1133-1141, November 1999.

[2] S. Srinivasan, G. Venkataramanan, "Comparative evaluation of PWM AC-AC converters," IEEE Power Electronic Specialist Conference, PESC, vol. 1, pp. 529-535, June 1995.

[3] H. Krishnaswami, V. Ramanarayanan, "Control of high frequency ac link electronic transformer," IEE Electric Power Applications, vol. 152 , no. 3, pp. 509- 516, May 2005.

[4] E.R. Ronan, S.D. Sudhoff, S.F. Glover, and D.L. Galloway, “A powerelectronic-based distribution transformer," IEEE Trans. on PowerDelivery., vol.17, pp. 537 - 543, April 2002.

[5] M. Sabahi, S. H. Hosseini, M. B. BannaeSharifian, A. YazdanpanahGoharrizi, G. B. Gharehpetian, "Three-Phase Dimmable Lighting System Using a Bidirectional Power Electronic Transformer,” IEEE Trans. Power Electronics, vol. 24, no. 3, pp. 830-837, MARCH 2009.

[6] H. Iman-eini, Sh. Farhangi, "Analysis and design of power electronic transformer for medium voltage levels," IEEE Power Electronic Specialist Conference, PESC, pp.1- 5, June 2006.

[7] H. Iman-Eini, JL. Schanen, Sh. Farhangi, J. Barbaroux, JP. Keradec, “APower Electronic Based Transformer for Feeding Sensitive Loads,’IEEE Power Electronics Specialists Conference,. PESC 2008, pp. 2549 -2555, 2008.

[8] D. Wang, C. Mao, J. Lu, S. Fan, F.Z. Peng, “Theory and application of distribution electronic power transformer," Electric. Power Syst. Res, vol. 77, pp. 219-226, March 2007.

[9] D. Wang, C. Mao, J. Lu, "Coordinated control of EPT and generator excitation system for multidouble-circuit transmission-lines system,’IEEE Trans. Power Deliver. vol. 23, no.1, pp. 371-379, 2008

[10] Keliang Zhou and Danwei Wang, Member, IEEE "Relationship Between Space-Vector Modulation and Three-Phase Carrier-Based PWM: A Comprehensive Analysis" VOL. 49, NO. 1, FEBRUARY 2002

[11] Dorin O. Neacsu "SPACE VECTOR MODULATION -An Introduction" The 27th Annual Conference of the IEEE Industrial Electronics Society

[12] Chuan-Sheng Liu *, Jonq-Chin Hwang **, and Po-Cheng, Chen *" Design of Permanent Magnet Synchronous Motor with low cogging torque" The 2010 International Power Electronics Conference.

\section{Authors Profile}

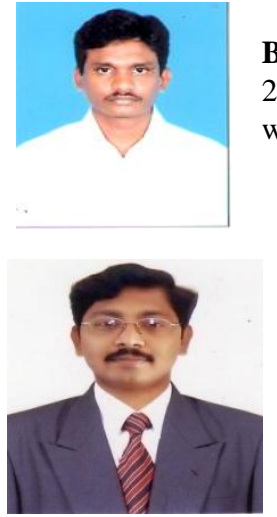

B.T.Kalyan has received his B.Tech degree (EEE) from Sri vasavi College Of Engg \& Tech in the year 2008. At present he is pursuing his Masters degree from the Swarnandhra College of engineering with the specialization of Power Electronics and Drives.

P.RAMPRASAD has received his B.Tech degree (EEE) from S.V.H.College of Engg\&Tech in the year 2004. He has received his M.Tech degree from Karunya University with the specialization of Power Electronics \& Drives in the year of 2009. His area of interest is Power Electronics,Industrial Drives. 